\title{
The Role of Procalcitonin in Predicting Risk of Mechanical Ventilation and Mortality among moderate to Severe COVID-19 Patients
}

Cher Wei Twe

Hospital Sungai Buloh

Delton Khoo

Hospital Sungai Buloh

Kian Boon Law

Institute for Clinical Research

Nur Sabreena Ahmad Nordin

Hospital Sungai Buloh

Subashini Sathasivan

Hospital Sungai Buloh

Kah Chuan Lim ( $\nabla$ ck7902@gmail.com )

Hospital Sungai Buloh

Sharifah Khairul Atikah

Hospital Sungai Buloh

Syarifah Nurul Ain Syed Badaruddin

Hospital Sungai Buloh

Suresh Kumar Chidambaram

Hospital Sungai Buloh

\section{Research Article}

Keywords: COVID-19, SARS-CoV-2, risk factor, procalcitonin, mechanical ventilation, mortality

Posted Date: November 8th, 2021

DOI: https://doi.org/10.21203/rs.3.rs-1026293/v1

License: (1) (i) This work is licensed under a Creative Commons Attribution 4.0 International License. Read Full License

Version of Record: A version of this preprint was published at BMC Infectious Diseases on April 15th, 2022. See the published version at https://doi.org/10.1186/s12879-022-07362-x. 


\section{Abstract}

\section{Background}

Serum procalcitonin (PCT) has become an emerging prognostic biomarker of disease progression in patients with COVID-19. This study aims to determine the optimal cut-off value of PCT with regards to important clinical outcomes, especially for mechanical ventilation and all-cause mortality among moderate to severe COVID-19 patients in Malaysia.

\section{Methods}

A total of 319 moderate to severe COVID-19 patients hospitalized at the National Referral Hospital in December 2020 were included in the study retrospectively. Demographics, comorbidities, the severity of COVID-19 infection, laboratory and imaging findings, and treatment given were collected from the hospital information system for analysis. The optimal cut-point values for PCT were estimated in two levels. The first level involved 276 patients who had their PCT measured within 5 days following their admission. The second level involved 237 patients who had their PCT measured within 3 days following their admission. Further, a propensity score matching analysis was performed to determine the adjusted relative risk of patients with regards to various clinical outcomes according to the selected cut-point among 237 patients who had their PCT measured within 3 days.

\section{Results}

The results showed that a PCT level of $0.2 \mathrm{ng} / \mathrm{mL}$ was the optimal cut-point for prognosis especially for mortality outcome and the need for mechanical ventilation. Before matching, patients with PCT $\geq$ $0.2 \mathrm{ng} / \mathrm{mL}$ were associated with significantly higher odds in all investigated outcomes. After matching, patients with PCT $>0.2 \mathrm{ng} / \mathrm{mL}$ were associated with higher odds in all-cause mortality (OR: $4.629,95 \% \mathrm{Cl}$ : $1.387-15.449, \mathrm{p}=0.0127$ ) and non-invasive ventilation (OR: 2.667, 95\% Cl: $1.039-6.847, \mathrm{p}=0.0415)$. Furthermore, patients with higher PCT were associated with significantly longer days of mechanical ventilation ( $p=0.0213)$. There was however no association between higher PCT level and the need for mechanical ventilation (OR: 2.010, 95\% Cl: $0.828-4.878, p=0.1229)$.

\section{Conclusion}

Our study indicates that a rise in PCT above $0.2 \mathrm{ng} / \mathrm{ml}$ is associated with an elevated risk in all-cause mortality, the need for non-invasive ventilation, and a longer duration of mechanical ventilation. The study offers concrete evidence for PCT to be used as a prognostication marker among moderate to severe COVID-19 patients.

\section{Background}

Since the outbreak of coronavirus disease 2019 (COVID-19) in December 2019 in Wuhan, China, the COVID-19 has rapidly spread across the world and was declared as a "Public Health Emergency of 
International Concern" on 30th January 2020. Then, it emerged as an unanticipated threat to global health and led the World Health Organisation (WHO) to further declare COVID-19 as a pandemic on 11th March $2020^{1}$. As of 18th September 2021, there are more than 226 million confirmed COVID-19 cases with 4.7 million deaths reported globally. Malaysia has reported 2,049,750 cases with 22,355 deaths on the same day ${ }^{2}$.

The clinical spectrum of COVID-19 ranges from asymptomatic to symptomatic severe disease with multiorgan involvement and failure requiring intensive care (ICU) admission and mechanical ventilation ${ }^{3}$. The broad clinical spectrum and highly variable clinical course among different COVID-19 patients have introduced great challenges in predicting the disease progression and outcome ${ }^{4}$. Laboratory biomarkers have always been very useful in day-to-day clinical practice to guide disease management and treatment decisions, especially in the management of infectious diseases like the COVID-19. Hence, ever since the start of the outbreak, the use of laboratory biomarkers including C-reactive protein (CRP), D-dimer, lactate dehydrogenase (LDH), and procalcitonin (PCT) to predict disease progression and severity have been explored and investigated extensively ${ }^{1,5}$.

Procalcitonin (PCT) is a 116-amino-acid peptide that shares a common molecular structure with the prohormone of calcitonin. It was first discovered in humans in 1981 by J.Allison et al but its clinical use was never really explored until 1993 when M.Assicot et al first suggested a positive association between elevated serum PCT and bacterial infection and sepsis ${ }^{6,7}$. In today's clinical practice, serum PCT has been increasingly instrumental in antibiotic stewardship and the diagnosis and management of sepsis due to bacterial infection ${ }^{8}$.

Since the beginning of the COVID-19 pandemic, multiple observational studies and meta-analyses have been done to look into the utility of serum PCT level as a biomarker of clinical deterioration among COVID-19 patients and have shown encouraging results with various optimal PCT cut-points suggested ${ }^{9-}$ 11. A single-center retrospective study done in Wuhan, China showed that a serum PCT level of more than $0.2 \mathrm{ng} / \mathrm{ml}$ was found in severe and critical COVID-19 patients ${ }^{12}$. Another retrospective study of 1099 patients reported that a PCT of more than $0.5 \mathrm{ng} / \mathrm{dL}$ was associated with increased severity of COVID-19 infection ${ }^{13}$. Theoretically, PCT, an acute phase peptide, is released in response to proinflammatory cytokines like IL-1 $\beta$, IL-6, and TNF- $\alpha$ typically induced by bacterial infection and are not seen in viral infections ${ }^{14}$. However, it was found that these pro-inflammatory cytokines are also raised in COVID-19 infection, especially among severe diseases, which makes serum PCT a promising biomarker of clinical deterioration among COVID-19 patients ${ }^{15}$.

This study aims to investigate the optimal cut-point of PCT and its relationship with regards to various clinical outcomes, especially in all-cause mortality and the need for mechanical ventilation among moderate to severe COVID-19 patients requiring hospitalization and treatment.

\section{Methods}




\section{Study design and setting}

A total of 319 patients who were diagnosed with moderate to severe COVID-19 pneumonia and admitted to Sungai Buloh Hospital in December 2020 were included and studied retrospectively. Demographic information, including age, gender, race, concomitant medical illnesses, the severity of COVID-19 infection, laboratory and imaging findings, and treatment given were retrieved from the hospital information system for analysis. The study included category 4 and 5 adult patients above 18 years old with a diagnosis of COVID-19 confirmed via polymerase chain reaction (PCR) test (Table 1). Patients were excluded if no reported PCT values.

The majority of patients (87.1\%) had their PCT levels measured within 5 days following their admission. As PCT levels may reduce over time after receiving treatment, we included patients with their PCT levels measured within 5 days and 3 days for the analysis of the optimal cut-point. Hence, the analysis of the optimal cut-point for PCT was performed in two levels. The first level involved 276 patients with their PCT levels measured within 5 days following admission and the second level involved 237 patients with their PCT levels measured within 3 days following admission. Besides, the study excluded patients with extremely high PCT levels (>100 ng/mL) to avoid overly skewed findings. Clinical outcomes included in the analysis for the optimal PCT cut-point were all-cause mortality, mechanical ventilation, the occurrence of thrombotic events, ICU admission, and bacterial infection. Figure 1 summarizes the patient recruitment process.

In the study, COVID-19 infection was classified according to the Malaysian Clinical Practice Guideline for Management of COVID-19 ${ }^{3}$. The guideline classifies COVID-19 severity into five clinical stages as shown in Table 1. Stage 1 and 2 consist of patients with asymptomatic infection or symptomatic infection with no pneumonia. Patients in these two categories are considered to have mild disease. Patients with stage 3 disease have lung involvement or pneumonia, but have not experienced hypoxemia, while stage 4 and 5 consist of critically ill patients requiring oxygen support and often intensive care. 


\begin{tabular}{|c|c|c|}
\hline $\begin{array}{l}\text { Clinical } \\
\text { Stage }\end{array}$ & Severity & Description \\
\hline \multirow[t]{2}{*}{1} & \multirow[t]{5}{*}{ Mild } & Asymptomatic \\
\hline & & - Only RT-PCR test positive \\
\hline \multirow[t]{3}{*}{2} & & Symptomatic, but no pneumonia \\
\hline & & $\begin{array}{l}\text { - Upper respiratory tract (URT) symptoms (e.g., pharyngeal congestion, sore } \\
\text { throat, cough, or fever) }\end{array}$ \\
\hline & & $\begin{array}{l}\text { - Other symptoms, like vomiting, diarrhea, abdominal pain, myalgia, loss of } \\
\text { smell/taste }\end{array}$ \\
\hline 3 & Moderate & Symptomatic, pneumonia, but no hypoxemia \\
\hline \multirow[t]{3}{*}{4} & \multirow[t]{3}{*}{ Severe } & Symptomatic, pneumonia, requiring supplemental oxygen \\
\hline & & OR \\
\hline & & $\begin{array}{l}\text { New requirement of supplemental oxygen or increased requirement from } \\
\text { baseline without need for non-invasive or invasive ventilation }\end{array}$ \\
\hline \multirow[t]{3}{*}{5} & \multirow[t]{3}{*}{ Critical } & Critically III with multiorgan involvement \\
\hline & & OR \\
\hline & & $\begin{array}{l}\text { New or increased need for non-invasive or invasive ventilation, including high } \\
\text { flow nasal cannula }\end{array}$ \\
\hline
\end{tabular}

\section{Analysis Of Pct}

In Sungai Buloh Hospital, serum PCT was measured by SIEMENS ATELLICA IMMUNOASSAY (IM). Atellica IM BRAHMS PCT assay is a 2-site sandwich immunoassay using direct chemiluminescent technology that uses 3 mouse monoclonal antibodies specific for PCT. The first antibody, in the Lite Reagent, is a mouse monoclonal anti-PCT antibody labeled with acridinium ester. The second and third antibodies, in the Ancillary Reagent, are mouse monoclonal anti-PCT antibodies labeled with fluorescein. The immunocomplex formed with PCT is captured with mouse monoclonal anti-fluorescein antibody coupled to paramagnetic particles in the Solid Phase. This 18-minute sandwich immunoassay with a measuring range of 0.03 to $50.00 \mathrm{ng} / \mathrm{mL}$, is aligned to the $B \cdot R \cdot A \cdot H \cdot M \cdot S$ PCT sensitive KRYPTOR® assay ${ }^{16}$.

\section{Statistical analysis}

Demographics and clinical characteristics were summarized in Table 2 for overall 319 patients, 276 and 237 patients who had their PCT measured within 5 days and 3 days following their admission. The 
optimal PCT cut-points were estimated using the receiver operative characteristic (ROC) method in two levels. In the first level, we included 276 patients who had their PCT levels measured within 5 days following their admission. In the second level, we included 237 patients who had their PCT levels measured within 3 days following their admission. The optimal cut-point was determined with priority on all-cause mortality and the need for mechanical ventilation. In further analysis, patients with their PCT levels measured within 3 days following admission were divided into two groups according to the selected cut-point. Patients with PCT levels above the cut-point were assumed to have a higher risk for inferior clinical outcomes, and vice versa. We compared and tested the differences of demographics, clinical characteristics, baseline laboratory findings, and co-morbidity profile between the two groups to identify covariates for adjustment in the propensity score matching analysis. The odds ratio (OR) was calculated and reported for various clinical outcomes with regards to the selected cut-point for PCT before and after the matching analysis. Independent t-test and Mann-Whitney $\mathrm{U}$ test were used to test differences of continuous variables between two groups, while Chi-squared test and Fisher's exact test were used to assess categorical variables. All statistical tests were performed at two-sided $5 \%$ significance level.

In propensity score matching analysis, patients were matched for covariates that were statistically different. Baseline laboratory parameters and vital signs were not included in the matching analysis as they were not inherited but clinical manifestations of COVID-19 infection or complications. Propensity score matching was done based on the nearest-neighbor method within a caliper width equal to 0.25 times the SD of the logit of the calculated propensity score. All analyses were performed in IBM SPSS version 26.0 for Windows and R version 4.1.0 with packages such as "cutpointr" and "Matchlt"17-19.

\section{Results}

Demographics, clinical characteristics, and clinical outcomes of patients were summarized in Table 2 according to the overall cohort, first and second level cohorts. Even with the exclusion of patients due to delayed measurement of PCT, first and second level cohorts were still similar to the overall cohort in terms of clinical characteristics, comorbidities profile, and clinical outcomes. 
Table 2

Demographics and clinical characteristics of the study cohort.

\begin{tabular}{|c|c|c|c|}
\hline Characteristics & $\begin{array}{l}\text { Overall } \\
\text { n (\%) }\end{array}$ & $\begin{array}{l}\text { First level } \\
\text { n (\%) }\end{array}$ & $\begin{array}{l}\text { Second } \\
\text { level } \\
\text { n (\%) }\end{array}$ \\
\hline Total & $\begin{array}{l}319 \\
(100.0)\end{array}$ & $\begin{array}{l}276 \\
(100.0)\end{array}$ & $237(100.0)$ \\
\hline Age, years: Mean (SD) & $56(13.6)$ & $54.8(13.7)$ & $55.0(13.7)$ \\
\hline Gender: Female & $111(34.8)$ & $102(37.0)$ & $84(35.4)$ \\
\hline \multicolumn{4}{|l|}{ Race } \\
\hline Malay & $168(52.7)$ & $147(53.3)$ & $122(51.5)$ \\
\hline Chinese & $68(21.3)$ & $54(19.6)$ & $46(19.4)$ \\
\hline Indian & $52(16.3)$ & $48(17.4)$ & $44(18.6)$ \\
\hline Others & $31(9.7)$ & $27(9.8)$ & $25(10.5)$ \\
\hline \multicolumn{4}{|l|}{ Comorbidities profile } \\
\hline Hypertension: Yes & $179(56.1)$ & $158(57.2)$ & $135(57.0)$ \\
\hline Chronic Cardiac Disease: Yes & $64(20.1)$ & $54(19.6)$ & $46(19.4)$ \\
\hline Chronic Pulmonary Disease: Yes & $10(3.1)$ & $8(2.9)$ & $7(3.0)$ \\
\hline Asthma: Yes & $14(4.4)$ & $13(4.7)$ & $10(4.2)$ \\
\hline Diabetes Mellitus: Yes & $147(46.1)$ & $128(46.4)$ & $110(46.4)$ \\
\hline Pre-existing Renal Disease: Yes & $73(22.9)$ & $56(20.3)$ & $46(19.4)$ \\
\hline Chronic Liver Disease: Yes & $3(0.9)$ & $2(0.7)$ & $2(0.8)$ \\
\hline Dementia: Yes & $3(0.9)$ & $3(1.1)$ & $2(0.8)$ \\
\hline Chronic Neurological Conditions: Yes & $16(5.0)$ & $13(4.7)$ & $10(4.2)$ \\
\hline Connective Tissue Disease: Yes & $7(2.2)$ & $7(2.5)$ & $5(2.1)$ \\
\hline HIV/AIDS: Yes & $2(0.6)$ & $1(0.4)$ & $1(0.4)$ \\
\hline Malignancy: Yes & $11(3.4)$ & $9(3.3)$ & $8(3.4)$ \\
\hline Current Smoking: Yes & $9(2.8)$ & $5(1.8)$ & $4(1.7)$ \\
\hline Obesity: Yes & $17(5.3)$ & $17(6.2)$ & $16(6.8)$ \\
\hline Others: Yes & $81(25.4)$ & $67(24.3)$ & $51(21.5)$ \\
\hline
\end{tabular}




\begin{tabular}{|c|c|c|c|}
\hline Characteristics & $\begin{array}{l}\text { Overall } \\
\text { n (\%) }\end{array}$ & $\begin{array}{l}\text { First level } \\
\text { n (\%) }\end{array}$ & $\begin{array}{l}\text { Second } \\
\text { level } \\
\text { n (\%) }\end{array}$ \\
\hline Category 4 & $254(79.6)$ & $217(78.6)$ & $181(76.4)$ \\
\hline Category 5 & $65(20.4)$ & $59(21.4)$ & $56(23.6)$ \\
\hline \multicolumn{4}{|l|}{ 4C mortality score } \\
\hline 0-3 (low-risk in-hospital mortality) & $16(5.0)$ & $15(5.4)$ & $13(5.5)$ \\
\hline 4-8 (intermediate-risk in-hospital mortality) & $117(36.7)$ & $105(38.0)$ & $87(36.7)$ \\
\hline 9-14 (high-risk in-hospital mortality) & $145(45.5)$ & $124(44.9)$ & $107(45.1)$ \\
\hline 15-21 (very high-risk in-hospital mortality) & $41(12.8)$ & $32(11.6)$ & $30(12.7)$ \\
\hline Steroid use: Yes & $297(93.1)$ & $257(93.1)$ & $219(92.4)$ \\
\hline Bloodstream infection: Yes & $35(11.0)$ & $28(13.0)$ & $25(13.4)$ \\
\hline \multicolumn{4}{|l|}{ Clinical outcomes } \\
\hline ICU admission: Yes & $143(44.8)$ & $129(46.7)$ & $120(50.6)$ \\
\hline NIV use: Yes & $65(20.4)$ & $60(21.7)$ & $57(24.1)$ \\
\hline Duration of NIV use, days, median (IQR) & $3(1.8,5.3)$ & $3(1.8,5.3)$ & $3(1,5)$ \\
\hline Mechanical ventilation: Yes & $101(31.7)$ & $89(32.2)$ & $85(35.9)$ \\
\hline $\begin{array}{l}\text { Duration of mechanical ventilation use, days, median } \\
\text { (IQR) }\end{array}$ & $6(4,12)$ & $7(4,12)$ & $7(4,12)$ \\
\hline \multicolumn{4}{|l|}{ Organizing pneumonia } \\
\hline Yes & $135(42.3)$ & $118(42.8)$ & $102(43.0)$ \\
\hline No & $26(8.2)$ & $21(7.6)$ & $19(8.0)$ \\
\hline CT scan not done & $158(49.5)$ & $137(49.6)$ & $116(48.9)$ \\
\hline Thrombotic event: Yes & $83(26.0)$ & $69(25.0)$ & $62(26.2)$ \\
\hline All-cause mortality: Yes & $71(22.3)$ & $59(21.4)$ & $55(23.2)$ \\
\hline Due to severe COVID-19 pneumonia & $48(67.6)$ & $43(72.9)$ & $39(70.9)$ \\
\hline Due to thrombotic event & $8(11.3)$ & $7(16.3)$ & $7(17.9)$ \\
\hline Due to comorbid & $7(9.8)$ & $4(9.3)$ & $4(10.3)$ \\
\hline Due to bacterial infections & $8(11.3)$ & $5(11.6)$ & $5(12.8)$ \\
\hline Bacteremia & $3(4.2)$ & $1(2.3)$ & $1(2.6)$ \\
\hline
\end{tabular}




\begin{tabular}{|llll|}
\hline Characteristics & $\begin{array}{l}\text { Overall } \\
\mathbf{n}(\%)\end{array}$ & $\begin{array}{l}\text { First level } \\
\mathbf{n}(\%)\end{array}$ & $\begin{array}{l}\text { Second } \\
\text { level } \\
\mathbf{n}(\%)\end{array}$ \\
\hline Intra-abdominal infection & $1(1.4)$ & $0(0)$ & $0(0)$ \\
\hline Necrotizing fasciitis & $1(1.4)$ & $1(2.3)$ & $1(2.6)$ \\
\hline Non-specified site & $1(1.4)$ & $0(0)$ & $0(0)$ \\
\hline Non-bacteremia & $5(7.0)$ & $4(9.3)$ & $4(10.3)$ \\
\hline Pulmonary infection & $2(2.8)$ & $1(2.3)$ & $1(2.6)$ \\
\hline Intra-abdominal infection & $1(1.4)$ & $1(2.3)$ & $1(2.6)$ \\
\hline Non-specified site & $2(2.8)$ & $2(4.7)$ & $2(5.1)$ \\
\hline
\end{tabular}

Column percentages are reported in parenthesis for categorical variables; mean and standard deviation are reported for continuous variable age; median and interquartile range are reported for continuous variables duration of non-invasive ventilation and mechanical ventilation.

Abbreviations: CT, computed tomography; HIV/AIDS, human immunodeficiency virus/acquired immunodeficiency syndrome; ICU; intensive care unit; NIV, non-invasive ventilation.

\section{Determination of the optimal cut-point}

In the determination of optimal cut-points for PCT, we included clinical outcomes, such as all-cause mortality, mechanical ventilation, thrombotic events, ICU admissions, and bacterial infection among moderate to severe COVID-19 patients. Table 3 and Table 4 present various performance indexes and optimal cut-points for PCT with regards to the aforementioned clinical outcomes based on PCT levels measured within 5 days and 3 days following admission.

For PCT levels measured within 5 days following admission, the optimal cut-points for PCT were found to be $0.20 \mathrm{ng} / \mathrm{mL}$ for all-cause mortality, $0.33 \mathrm{ng} / \mathrm{mL}$ for mechanical ventilation, $1.21 \mathrm{ng} / \mathrm{mL}$ for thrombotic events, $0.09 \mathrm{ng} / \mathrm{mL}$ for ICU admission dan $1.2 \mathrm{ng} / \mathrm{mL}$ for bacterial infection (Table 3). Highest sensitivity was found in all-cause mortality (0.8136) for PCT cut-point of $0.20 \mathrm{ng} / \mathrm{mL}$. For PCT levels measured within 3 days following admission, the optimal cut-points for PCT were found to be $0.20 \mathrm{ng} / \mathrm{mL}$ for allcause mortality, $0.21 \mathrm{ng} / \mathrm{mL}$ for mechanical ventilation, $1.21 \mathrm{ng} / \mathrm{mL}$ for thrombotic events, $0.07 \mathrm{ng} / \mathrm{mL}$ for ICU admission dan $1.25 \mathrm{ng} / \mathrm{mL}$ for bacterial infection (Table 4). Highest sensitivity was also found in all-cause mortality (0.8364) for PCT cut-point of $0.20 \mathrm{ng} / \mathrm{mL}$.

As $0.2 \mathrm{ng} / \mathrm{mL}$ appeared to be the optimal cut-point in all-cause mortality for PCT levels measured within 5 days and 3 days following admission, and in mechanical ventilation for PCT levels measured within 3 days following admission, therefore, $0.2 \mathrm{ng} / \mathrm{mL}$ was selected as the final PCT cut-point in the matching analysis using propensity scores. The PCT of $0.2 \mathrm{ng} / \mathrm{mL}$ represented the optimal cut-point for the two 
clinically important outcomes, all-cause mortality and mechanical ventilation, especially for moderate to severe COVID-19 patients with PCT measured within 3 days following admission.

Table 3

Optimal cut-off PCT values (within 5 days from admission)

\begin{tabular}{|llllll|}
\hline $\begin{array}{l}\text { Cut-point and } \\
\text { Indexes }\end{array}$ & $\begin{array}{l}\text { All-cause } \\
\text { mortality }\end{array}$ & $\begin{array}{l}\text { Mechanical } \\
\text { ventilation }\end{array}$ & $\begin{array}{l}\text { Thrombotic } \\
\text { events }\end{array}$ & $\begin{array}{l}\text { ICU } \\
\text { admission }\end{array}$ & $\begin{array}{l}\text { Bacterial } \\
\text { infection } \\
\text { culture }\end{array}$ \\
\hline $\begin{array}{l}\text { Number of } \\
\text { patients }\end{array}$ & 276 & 276 & 276 & 276 & 216 \\
\hline Positive cases & 59 & 89 & 69 & 129 & 28 \\
\hline Negative cases & 217 & 187 & 207 & 147 & 188 \\
\hline AUC & 0.7741 & 0.7773 & 0.6666 & 0.7223 & 0.6444 \\
\hline $\begin{array}{l}\text { Optimal cut-point } \\
\text { (ng/ml) }\end{array}$ & 0.20 & 0.33 & 1.21 & 0.09 & 1.2 \\
\hline Accuracy & 0.6703 & 0.7391 & 0.7536 & 0.6848 & 0.7454 \\
\hline Sensitivity & 0.8136 & 0.6742 & 0.4203 & 0.7442 & 0.5357 \\
\hline Specificity & 0.6313 & 0.7701 & 0.8647 & 0.6327 & 0.7766 \\
\hline Precision & 0.3750 & 0.5825 & 0.5088 & 0.6400 & 0.2632 \\
\hline TP & 48 & 60 & 29 & 96 & 15 \\
\hline FN & 11 & 29 & 40 & 33 & 13 \\
\hline FP & 54 & 43 & 28 & 43 & 42 \\
\hline TN & 93 & 144 & 179 & 144 & 146 \\
\hline TP: True positive; FN: False negative; FP: False positive; TN: True negative; AUC: Area under Curve \\
\hline
\end{tabular}


Table 4

Optimal cut-off PCT values (within 3 days from admission)

\begin{tabular}{|llllll|}
\hline $\begin{array}{l}\text { Cut-point and } \\
\text { Indexes }\end{array}$ & $\begin{array}{l}\text { All-cause } \\
\text { mortality }\end{array}$ & $\begin{array}{l}\text { Mechanical } \\
\text { ventilation }\end{array}$ & $\begin{array}{l}\text { Thrombotic } \\
\text { events }\end{array}$ & $\begin{array}{l}\text { ICU } \\
\text { admission }\end{array}$ & $\begin{array}{l}\text { Bacterial } \\
\text { infection } \\
\text { culture }\end{array}$ \\
\hline $\begin{array}{l}\text { Number of } \\
\text { patients }\end{array}$ & 237 & 237 & 237 & 237 & 186 \\
\hline Positive cases & 55 & 85 & 62 & 120 & 25 \\
\hline Negative cases & 182 & 152 & 175 & 117 & 161 \\
\hline AUC & 0.7812 & 0.7676 & 0.6711 & 0.7365 & 0.6465 \\
\hline $\begin{array}{l}\text { Optimal cut-point } \\
\text { (ng/ml) }\end{array}$ & 0.20 & 0.21 & 1.21 & 0.07 & 1.25 \\
\hline Accuracy & 0.6667 & 0.7046 & 0.7468 & 0.7046 & 0.7419 \\
\hline Sensitivity & 0.8364 & 0.7528 & 0.4355 & 0.8333 & 0.5600 \\
\hline Specificity & 0.6154 & 0.6776 & 0.8571 & 0.5726 & 0.7702 \\
\hline Precision & 0.3966 & 0.5663 & 0.5192 & 0.6667 & 0.2745 \\
\hline TP & 46 & 64 & 27 & 100 & 14 \\
\hline FN & 9 & 21 & 35 & 20 & 11 \\
\hline FP & 70 & 49 & 25 & 50 & 37 \\
\hline TN & 112 & 103 & 150 & 67 & 124 \\
\hline TP: True positive; FN: False negative; FP: False positive; TN: True negative; AUC: Area under Curve \\
\hline
\end{tabular}

\section{Propensity scores matching analysis}

To assess the prognostic value of the optimal cut-point, the analysis focused on those who had their PCT levels measured within 3 days following admission. Moreover, the selected cut-point of $0.2 \mathrm{ng} / \mathrm{mL}$ was optimal for all-cause mortality and mechanical ventilation among those with PCT levels measured within 3 days following admission. Table 5 summarizes the covariate differences according to the selected optimal cut-point for PCT among 237 patients. Significant differences were identified for age $(p=0.0122)$, COVID-19 disease stage $(p<0.0001)$, days of illness before admission $(\mathrm{p}=0.0036), \mathrm{SPO}_{2}$ under room temperature (RA) ( $p=0.0005)$, Glasgow Coma Scale (GCS) $(p<0.0001)$, urea $(p<0.0001)$, C-reactive protein (CRP) $(p<0.0001)$, white blood cells count (WBC) $(p=0.0003)$, absolute neutrophil count (ANC) $(p<0.0001)$, number of comorbidities $(p<0.0001)$, presence of hypertension $(p=0.0007)$, chronic cardiac diseases $(p=0.0018)$, diabetes mellitus $(D M)(p=0.0036)$, pre-existing renal disease $(p<0.0001)$ and malignancy $(p=0.0327)$. 
Table 6 presents the odds ratio (OR) with regards to various clinical outcomes based on the optimal cutpoint of $0.2 \mathrm{ng} / \mathrm{mL}$ for PCT. Prior to matching, our analysis found that patients with PCT level above 0.2 $\mathrm{ng} / \mathrm{mL}$ were associated with significantly higher risk in all-cause mortality (OR: 8.178, 95\%Cl: 3.770 17.738, $p<0.0001$ ), mechanical ventilation (OR: $5.861,95 \% \mathrm{Cl}: 3.229-10.637, p<0.0001$ ), non-invasive ventilation (OR: $2.898,95 \% \mathrm{Cl}: 1.541$ - 5.541, $\mathrm{p}=0.0010$ ), ICU admission (OR: 4.166, 95\%Cl: 2.422 - 7.166, $p<0.0001$ ) and thrombotic events (OR: $2.158,95 \% \mathrm{Cl}: 1.190-3.915, p=0.0013)$. Besides, patients with PCT levels above $0.2 \mathrm{ng} / \mathrm{mL}$ also experienced significantly longer days of mechanical ventilation $(p<0.0106)$ and length of hospital stay $(p<0.0001)$. Table 7 shows the mortality and causes of death before propensity score matching. 
Table 5

Demographics, clinical characteristics \& baseline laboratory findings, and comorbidity profile of COVID-19 patients according to procalcitonin level.

\begin{tabular}{|c|c|c|c|c|}
\hline \multirow[t]{2}{*}{ Variables } & \multirow[t]{2}{*}{ Category } & \multirow{2}{*}{$\begin{array}{l}\mathrm{PCT}<0.2 \\
(\mathrm{ng} / \mathrm{ml}) \\
(\mathrm{n}=121)\end{array}$} & \multirow{2}{*}{$\begin{array}{l}\mathrm{PCT} \geq 0.2 \\
(\mathrm{ng} / \mathrm{ml})\end{array}$} & \multirow[t]{2}{*}{ p-value } \\
\hline & & & & \\
\hline \multicolumn{5}{|l|}{ Demographics } \\
\hline Gender & Female & $42(34.7)$ & $42(36.2)$ & 0.8097 \\
\hline Age (years) & $\begin{array}{l}\text { Mean } \\
\text { (SD) }\end{array}$ & $52.8(12.6)$ & $57.3(14.4)$ & 0.0122 \\
\hline \multirow[t]{4}{*}{ Age groups (years) } & $<50$ & $45(37.2)$ & $33(28.4)$ & 0.0945 \\
\hline & $50-59$ & $38(31.4)$ & $28(24.1)$ & \\
\hline & $60-60$ & $23(19.0)$ & $34(29.3)$ & \\
\hline & $\geq 70$ & $15(12.4)$ & $21(18.1)$ & \\
\hline \multirow[t]{4}{*}{ Ethnicity } & Malay & $60(62.3)$ & $62(53.4)$ & 0.0675 \\
\hline & Chinese & $22(18.2)$ & $24(20.7)$ & \\
\hline & Indian & $20(16.5)$ & $24(20.7)$ & \\
\hline & Others & $19(15.7)$ & $6(5.2)$ & \\
\hline \multicolumn{5}{|c|}{ Clinical characteristics \& baseline laboratory } \\
\hline \multirow[t]{2}{*}{ Severity of disease } & $\begin{array}{l}\text { Category } \\
4\end{array}$ & $111(91.7)$ & $70(60.3)$ & $\dot{0} 0001$ \\
\hline & $\begin{array}{l}\text { Category } \\
5\end{array}$ & $10(8.3)$ & $46(39.7)$ & \\
\hline Days of illness & $\begin{array}{l}\text { Mean } \\
\text { (SD) }\end{array}$ & $6.5(3.0)$ & $5.3(3.1)$ & 0.0036 \\
\hline \multirow[t]{2}{*}{ SPO2 under RA (\%) } & $<92 \%$ & $98(81.0)$ & $111(95.7)$ & 0.0005 \\
\hline & $\geq 92 \%$ & $23(19.0)$ & $5(4.3)$ & \\
\hline \multirow[t]{3}{*}{$\mathrm{RR}, \mathrm{bpm}$} & $<20$ & $13(10.7)$ & $14(12.1)$ & 0.2520 \\
\hline & $20-29$ & $93(76.9)$ & $79(68.1)$ & \\
\hline & $\geq 30$ & $15(12.4)$ & $23(19.8)$ & \\
\hline GCS & $<15$ & $11(9.1)$ & $50(43.1)$ & $<0.0001$ \\
\hline
\end{tabular}

Abbreviations: ANC, absolute neutrophil count; CRP, C-reactive protein; GCS, Glasgow coma scale; HIV/AIDS, human immunodeficiency virus/acquired immunodeficiency syndrome; PCT, procalcitonin; RR, respiratory rate; RA, room air; SD, standard deviation; WBC, white blood cell count. 


\begin{tabular}{|c|c|c|c|c|}
\hline \multirow[t]{2}{*}{ Variables } & \multirow[t]{2}{*}{ Category } & \multirow{2}{*}{$\begin{array}{l}\mathrm{PCT}<0.2 \\
(\mathrm{ng} / \mathrm{ml}) \\
(\mathrm{n}=121)\end{array}$} & \multirow{2}{*}{$\begin{array}{l}\text { PCT } \geq 0.2 \\
(\mathrm{ng} / \mathrm{ml})\end{array}$} & \multirow[t]{2}{*}{ p-value } \\
\hline & & & & \\
\hline & 15 & $110(90.9)$ & $66(56.9)$ & \\
\hline \multirow[t]{3}{*}{ Urea, mmol/dL } & $<7$ & 87 (71.9) & $31(26.7)$ & $<0.0001$ \\
\hline & $7-14$ & $29(24.0)$ & $31(26.7)$ & \\
\hline & $>14$ & $5(4.1)$ & $54(46.6)$ & \\
\hline \multirow[t]{3}{*}{ CRP, mg/dL } & $<5$ & $49(40.5)$ & $8(6.9)$ & $<0.0001$ \\
\hline & $5-9.9$ & $31(25.6)$ & $69(59.5)$ & \\
\hline & $\geq 10$ & 41 (33.9) & $39(33.6)$ & \\
\hline WBC (x10^12/L) & $\begin{array}{l}\text { Mean } \\
\text { (SD) }\end{array}$ & $8.56(3.88)$ & $10.90(5.80)$ & 0.0003 \\
\hline ANC (x10^9/L) & $\begin{array}{l}\text { Mean } \\
\text { (SD) }\end{array}$ & $6.75(3.81)$ & $9.26(5.53)$ & $<0.0001$ \\
\hline \multicolumn{5}{|l|}{ Comorbidity profile } \\
\hline \multirow[t]{3}{*}{ No. of comorbidity } & 0 & $67(55.4)$ & $33(28.4)$ & $<0.0001$ \\
\hline & 1 & $42(34.7)$ & $33(28.4)$ & \\
\hline & $\geq 2$ & $12(9.9)$ & $50(43.1)$ & \\
\hline Hypertension & Yes & $56(46.3)$ & $79(68.1)$ & 0.0007 \\
\hline $\begin{array}{l}\text { Chronic cardiac disease (excluding } \\
\text { HPT) }\end{array}$ & Yes & $14(11.6)$ & $32(27.6)$ & 0.0018 \\
\hline Asthma & Yes & $6(5.0)$ & $4(3.4)$ & 0.7491 \\
\hline $\begin{array}{l}\text { Chronic pulmonary disease (excluding } \\
\text { asthma) }\end{array}$ & Yes & $3(2.5)$ & $4(3.4)$ & 0.7174 \\
\hline Diabetes Mellitus & Yes & $45(37.2)$ & $65(56.0)$ & 0.0036 \\
\hline Pre-existing renal disease & Yes & $5(4.1)$ & $41(35.3)$ & $<0.0001$ \\
\hline Stage 2 & & $2(40.0)$ & 0 & \\
\hline Stage 3 & & 0 & $6(14.6)$ & \\
\hline Stage 4 & & $2(40.0)$ & 8 (19.5) & \\
\hline
\end{tabular}

Abbreviations: ANC, absolute neutrophil count; CRP, C-reactive protein; GCS, Glasgow coma scale; HIV/AIDS, human immunodeficiency virus/acquired immunodeficiency syndrome; PCT, procalcitonin; RR, respiratory rate; RA, room air; SD, standard deviation; WBC, white blood cell count. 


\begin{tabular}{|c|c|c|c|c|}
\hline \multirow[t]{2}{*}{ Variables } & \multirow[t]{2}{*}{ Category } & \multirow{2}{*}{$\begin{array}{l}\text { PCT < } 0.2 \\
(\mathrm{ng} / \mathrm{ml}) \\
(\mathrm{n}=121)\end{array}$} & \multirow{2}{*}{$\begin{array}{l}\mathrm{PCT} \geq 0.2 \\
(\mathrm{ng} / \mathrm{ml})\end{array}$} & \multirow[t]{2}{*}{ p-value } \\
\hline & & & & \\
\hline Stage 5 & & $1(20.0)$ & $27(65.9)$ & \\
\hline HIV/AIDS & Yes & 1 & 0 & n.a \\
\hline Malignancy & Yes & $1(0.8)$ & $7(6.0)$ & 0.0327 \\
\hline Smoking & $\begin{array}{l}\text { Yes, } \\
\text { current }\end{array}$ & $1(0.8)$ & $3(2.6)$ & n.a \\
\hline Obesity $\left(>30 \mathrm{~kg} / \mathrm{m}^{2}\right)$ & Yes & $7(5.8)$ & $9(7.8)$ & 0.5449 \\
\hline Chronic liver disease & Yes & 0 & 2 & n.a \\
\hline Dementia & Yes & 0 & 2 & n.a \\
\hline Chronic neurological disease & Yes & $2(1.7)$ & $8(6.9)$ & 0.0555 \\
\hline Connective tissue disease & Yes & $2(1.7)$ & $3(2.6)$ & 0.6783 \\
\hline $\begin{array}{l}\text { Abbreviations: ANC, absolute } \\
\text { HIV/AIDS, human immunodef } \\
\text { RR, respiratory rate; RA, room }\end{array}$ & $\begin{array}{l}\text { unt; CRP, C } \\
\text { lacquired ir } \\
\text { ard deviati }\end{array}$ & $\begin{array}{l}\text { active prote } \\
\text { nunodefici } \\
\text {; WBC, whit }\end{array}$ & $\begin{array}{l}\text {, Glasgow c } \\
\text { ndrome; PCT } \\
\text { cell count. }\end{array}$ & $\begin{array}{l}\text { cale; } \\
\text { Icitonin; }\end{array}$ \\
\hline
\end{tabular}


Table 6

Clinical outcomes before propensity score matching.

\begin{tabular}{|c|c|c|c|c|c|}
\hline \multirow[t]{3}{*}{ Clinical outcomes } & \multirow[t]{3}{*}{ Category } & \multicolumn{2}{|c|}{ PCT level (ng/ml) } & \multirow[t]{3}{*}{ OR $(95 \% \mathrm{Cl})$} & \multirow{3}{*}{ p-value } \\
\hline & & РCT $<0.2$ & PCT $\geq 0.2$ & & \\
\hline & & $(n=121)$ & $(n=116)$ & & \\
\hline Procalcitonin level & $\begin{array}{l}\text { Mean } \\
\text { (SD) }\end{array}$ & $\begin{array}{l}0.0674 \\
(0.0331)\end{array}$ & $\begin{array}{l}6.002 \\
(11.556)\end{array}$ & n.a & $<0.0001$ \\
\hline \multirow[t]{2}{*}{ All-cause mortality } & Yes & $9(7.4)$ & $46(39.7)$ & $\begin{array}{l}8.178(3.770- \\
17.738)\end{array}$ & $<0.0001$ \\
\hline & No & $112(92.6)$ & $70(60.3)$ & & \\
\hline \multirow[t]{2}{*}{ Mechanical ventilation } & Yes & $21(17.4)$ & $64(55.2)$ & $\begin{array}{l}5.861(3.229- \\
10.637)\end{array}$ & $<0.0001$ \\
\hline & No & $100(82.6)$ & $52(44.8)$ & & \\
\hline \multirow[t]{2}{*}{ Non-invasive ventilation } & Yes & $18(14.9)$ & $39(33.6)$ & $\begin{array}{l}2.898(1.541- \\
5.541)\end{array}$ & 0.0010 \\
\hline & No & $103(85.1)$ & $77(66.4)$ & & \\
\hline \multirow[t]{2}{*}{ ICU admission } & Yes & $41(33.9)$ & $79(68.1)$ & $\begin{array}{l}4.166(2.422- \\
7.166)\end{array}$ & $<0.0001$ \\
\hline & No & $80(66.1)$ & 37 (31.9) & & \\
\hline \multirow[t]{2}{*}{ Thrombotic events } & Yes & $23(19.0)$ & $39(33.6)$ & $\begin{array}{l}2.158(1.190- \\
3.915)\end{array}$ & 0.0113 \\
\hline & No & $98(81.0)$ & $77(66.4)$ & & \\
\hline \multirow[t]{2}{*}{ Positive culture } & Yes & $68(89.5)$ & $93(84.5)$ & $\begin{array}{l}0.643(0.263- \\
1.578)\end{array}$ & 0.3354 \\
\hline & No & $8(10.5)$ & $17(15.5)$ & & \\
\hline $\begin{array}{l}\text { Days of mechanical } \\
\text { ventilation }\end{array}$ & $\begin{array}{l}\text { Median } \\
\text { (IQR) }\end{array}$ & $5(4-6)$ & $9(5-12.3)$ & n.a & 0.0106 \\
\hline $\begin{array}{l}\text { Days of non-invasive } \\
\text { ventilation }\end{array}$ & $\begin{array}{l}\text { Median } \\
\text { (IQR) }\end{array}$ & $3(2-4)$ & $3(1-6)$ & & 0.8071 \\
\hline $\begin{array}{l}\text { Length of hospital stay, } \\
\text { days }\end{array}$ & $\begin{array}{l}\text { Median } \\
\text { (IQR) }\end{array}$ & $11(9-14)$ & $\begin{array}{l}16(11.3- \\
20.8)\end{array}$ & n.a & $<0.0001$ \\
\hline
\end{tabular}


Table 7

Mortality and causes of death before propensity score matching

\begin{tabular}{|ll|}
\hline Mortality outcomes & Cases (\%) \\
\hline Total number of deaths & 55 \\
\hline Cause of death & \\
\hline Severe COVID-19 pneumonia & $39(70.9)$ \\
\hline Bacterial infection & $5(9.1)$ \\
\hline Bacteremia & 1 \\
\hline Non-bacteremia & 4 \\
\hline Thrombotic event & $7(12.7)$ \\
\hline Comorbidity & $4(7.3)$ \\
\hline
\end{tabular}

As the relative risk of patients categorized based on the optimal cut-point could be confounded by covariates that were significantly different, a propensity score matching was performed with the aforementioned covariates to derive two comparable arms for further analysis. The propensity score matching eventually identified 90 patients with balanced demographics, clinical characteristics, comorbidities profile, and laboratory findings except for urea $(p=0.0139)$ and CRP $(p<0.0001)$ as shown in Table 8. Subsequent analyses showed that patients with PCT above $0.2 \mathrm{ng} / \mathrm{mL}$ were associated with a significantly higher risk in all-cause mortality (OR: 4.629, 95\%Cl: $1.387-15.449, p=0.0127$ ), and marginally higher risk for non-invasive ventilation (OR: 2.667, 95\% Cl: $1.039-6.847, \mathrm{p}=0.0415)$. Besides, patients with PCT above $0.2 \mathrm{ng} / \mathrm{mL}$ tended to experience longer days of mechanical ventilation $(p=0.0213)$ as summarized in Table 9 . There was no significant association between PCT level and risk of mechanical ventilation (OR; $2.010,95 \% \mathrm{Cl} ; 0.828-4.878, \mathrm{p}=0.1229)$ after being matched for covariate differences. 
Table 8

Demographics, clinical characteristics \& baseline laboratory findings and comorbidity profile of COVID-19 patients according to procalcitonin level after propensity score matching

\begin{tabular}{|c|c|c|c|c|}
\hline \multirow[t]{2}{*}{ Variables } & \multirow[t]{2}{*}{ Category } & \multirow{2}{*}{$\begin{array}{l}\text { PCT }<0.2 \\
(n=45)\end{array}$} & \multirow{2}{*}{$\begin{array}{l}\mathrm{PCT} \geq 0.2 \\
(\mathrm{n}=45)\end{array}$} & \multirow[t]{2}{*}{ p-value } \\
\hline & & & & \\
\hline \multicolumn{5}{|l|}{ Demographics } \\
\hline Gender & Female & $17(37.8)$ & $17(37.8)$ & 1.0000 \\
\hline Age & Mean (SD) & $\begin{array}{l}55.1 \\
(11.2)\end{array}$ & $53.4(13.1)$ & 0.5070 \\
\hline \multirow[t]{4}{*}{ Age groups } & $<50$ & $13(28.9)$ & $16(35.6)$ & 0.7838 \\
\hline & $50-59$ & $14(31.1)$ & 15 (33.3) & \\
\hline & $60-60$ & $13(28.9)$ & $9(20.0)$ & \\
\hline & $\geq 70$ & $5(11.1)$ & $5(11.1)$ & \\
\hline \multirow[t]{4}{*}{ Ethnicity } & Malay & $22(48.9)$ & $27(60.0)$ & 0.6236 \\
\hline & Chinese & $9(20.0)$ & $7(15.6)$ & \\
\hline & Indian & $8(17.8)$ & $8(17.8)$ & \\
\hline & Others & $6(13.3)$ & $3(6.7)$ & \\
\hline \multicolumn{5}{|c|}{ Clinical characteristics \& baseline laboratory } \\
\hline \multirow[t]{2}{*}{ Severity of disease } & Category 4 & $35(77.8)$ & $37(82.2)$ & 0.5982 \\
\hline & Category 5 & $10(22.2)$ & $8(17.8)$ & \\
\hline Days of illness & Mean (SD) & $5.7(2.8)$ & $5.6(3.0)$ & 0.8852 \\
\hline \multirow[t]{2}{*}{ SPO2 under RA (\%) } & $<92 \%$ & $38(84.4)$ & $42(93.3)$ & 0.1797 \\
\hline & $\geq 92 \%$ & $7(15.6)$ & $3(6.7)$ & \\
\hline \multirow[t]{3}{*}{$\mathrm{RR}, \mathrm{bpm}$} & $<20$ & $5(11.1)$ & $4(8.9)$ & \\
\hline & $20-29$ & 33 (73.3) & $31(68.9)$ & \\
\hline & $\geq 30$ & $7(15.6)$ & $10(22.2)$ & \\
\hline \multirow[t]{2}{*}{ GCS } & $<15$ & $9(20.0)$ & $11(24.4)$ & 0.6121 \\
\hline & 15 & $36(80.0)$ & $34(75.6)$ & \\
\hline Urea, mmol/dL & $<7$ & $24(53.3)$ & $20(44.4)$ & 0.0139 \\
\hline \multicolumn{5}{|c|}{$\begin{array}{l}\text { Abbreviations: ANC, absolute neutrophil count; CRP, C-reactive protein; GCS, Glasgow Coma Scale; } \\
\text { HIV/AIDS, human immunodeficiency virus/acquired immunodeficiency syndrome; PCT, procalcitonin } \\
\text { RR, respiratory rate; RA, room air; SD, standard deviation; WBC, white blood cell count. }\end{array}$} \\
\hline
\end{tabular}




\begin{tabular}{|c|c|c|c|c|}
\hline \multirow[t]{2}{*}{ Variables } & \multirow[t]{2}{*}{ Category } & \multirow{2}{*}{$\begin{array}{l}\text { PCT }<0.2 \\
(n=45)\end{array}$} & \multirow{2}{*}{$\begin{array}{l}\text { PCT } \geq 0.2 \\
(n=45)\end{array}$} & \multirow[t]{2}{*}{ p-value } \\
\hline & & & & \\
\hline & $7-14$ & $17(37.8)$ & $10(22.2)$ & \\
\hline & $>14$ & $4(8.9)$ & 15 (33.3) & \\
\hline \multirow[t]{3}{*}{$\mathrm{CRP}, \mathrm{mg} / \mathrm{dL}$} & $<5$ & $17(37.8)$ & $1(2.2)$ & $<0.0001$ \\
\hline & $5-9.9$ & $13(28.9)$ & $15(33.3)$ & \\
\hline & $\geq 10$ & $15(33.3)$ & $29(64.4)$ & \\
\hline WBC (x10^12/L) & Mean (SD) & $\begin{array}{l}9.79 \\
(4.26)\end{array}$ & $\begin{array}{l}10.69 \\
(6.09)\end{array}$ & 0.4148 \\
\hline ANC (x10^9/L) & Mean (SD) & $\begin{array}{l}7.89 \\
(4.32)\end{array}$ & $8.74(5.82)$ & 0.4328 \\
\hline \multicolumn{5}{|l|}{ Comorbidity profile } \\
\hline \multirow[t]{3}{*}{ No. of comorbidity } & 0 & $15(33.3)$ & $15(33.3)$ & 0.9654 \\
\hline & 1 & $19(42.2)$ & $18(40.0)$ & \\
\hline & $\geq 2$ & $11(24.4)$ & $12(26.7)$ & \\
\hline Hypertension & Yes & $24(53.3)$ & $27(60.0)$ & 0.5234 \\
\hline Chronic cardiac disease (excluding HPT) & Yes & $12(26.7)$ & $12(26.7)$ & 1.0000 \\
\hline Asthma & Yes & $1(2.2)$ & 0 & n.a \\
\hline $\begin{array}{l}\text { Chronic pulmonary disease (excluding } \\
\text { asthma) }\end{array}$ & Yes & $1(2.2)$ & $2(4.4)$ & n.a \\
\hline Diabetes Mellitus & Yes & $24(53.3)$ & $21(46.7)$ & 0.5271 \\
\hline Pre-existing renal disease & Yes & $5(11.1)$ & $9(20.0)$ & 0.2447 \\
\hline Stage 2 & & 2 & 0 & \\
\hline Stage 3 & & 0 & 1 & \\
\hline Stage 4 & & 2 & 0 & \\
\hline Stage 5 & & 1 & 8 & \\
\hline HIV/AIDS & Yes & 0 & 0 & n.a \\
\hline Malignancy & Yes & $1(2.2)$ & 0 & n.a \\
\hline
\end{tabular}

Abbreviations: ANC, absolute neutrophil count; CRP, C-reactive protein; GCS, Glasgow Coma Scale; HIV/AIDS, human immunodeficiency virus/acquired immunodeficiency syndrome; PCT, procalcitonin; RR, respiratory rate; RA, room air; SD, standard deviation; WBC, white blood cell count. 


\begin{tabular}{|c|c|c|c|c|}
\hline \multirow[t]{2}{*}{ Variables } & \multirow[t]{2}{*}{ Category } & \multirow{2}{*}{$\begin{array}{l}\text { PCT }<0.2 \\
(n=45)\end{array}$} & \multirow{2}{*}{$\begin{array}{l}\text { PCT } \geq 0.2 \\
(n=45)\end{array}$} & \multirow[t]{2}{*}{ p-value } \\
\hline & & & & \\
\hline Smoking & $\begin{array}{l}\text { Yes, } \\
\text { current }\end{array}$ & 0 & $2(4.4)$ & n.a \\
\hline Obesity $\left(>30 \mathrm{~kg} / \mathrm{m}^{2}\right)$ & Yes & $2(4.4)$ & $4(8.9)$ & 0.6766 \\
\hline Chronic liver disease & Yes & 0 & $1(2.2)$ & \\
\hline Dementia & Yes & 0 & 0 & \\
\hline Chronic neurological disease & Yes & $2(4.4)$ & $1(2.2)$ & \\
\hline Connective tissue disease & Yes & $2(4.4)$ & $2(4.4)$ & \\
\hline \multicolumn{5}{|c|}{$\begin{array}{l}\text { Abbreviations: ANC, absolute neutrophil count; CRP, C-reactive protein; GCS, Glasgow Coma Scale; } \\
\text { HIV/AIDS, human immunodeficiency virus/acquired immunodeficiency syndrome; PCT, procalcitonin; } \\
\text { RR, respiratory rate; RA, room air; SD, standard deviation; WBC, white blood cell count. }\end{array}$} \\
\hline
\end{tabular}


Table 9

Clinical outcomes after propensity score matching.

\begin{tabular}{|c|c|c|c|c|c|}
\hline \multirow[t]{2}{*}{ Clinical outcomes } & \multirow[t]{2}{*}{ Category } & \multicolumn{2}{|l|}{ PCT level } & \multirow[t]{2}{*}{ OR $(95 \% \mathrm{Cl})$} & \multirow[b]{2}{*}{$\begin{array}{l}\mathrm{p}- \\
\text { value }\end{array}$} \\
\hline & & $\begin{array}{l}\text { РCT }<0.2 \\
(n=45)\end{array}$ & $\begin{array}{l}\text { РCT } \geq 0.2 \\
(n=45)\end{array}$ & & \\
\hline Procalcitonin level & $\begin{array}{l}\text { Mean } \\
\text { (SD) }\end{array}$ & $\begin{array}{l}0.068 \\
(0.033)\end{array}$ & $\begin{array}{l}4.849 \\
(11.624)\end{array}$ & n.a & 0.0084 \\
\hline \multirow[t]{2}{*}{ All-cause mortality } & Yes & $4(8.9)$ & $14(31.1)$ & $\begin{array}{l}4.629(1.387- \\
15.449)\end{array}$ & 0.0127 \\
\hline & No & $41(91.1)$ & $31(68.9)$ & & \\
\hline \multirow[t]{2}{*}{ Mechanical ventilation } & Yes & $12(26.7)$ & $19(42.2)$ & $\begin{array}{l}2.010(0.828- \\
4.878)\end{array}$ & 0.1229 \\
\hline & No & 33 (73.3) & $26(57.8)$ & & \\
\hline \multirow[t]{2}{*}{ Non-invasive ventilation } & Yes & $9(20.0)$ & $18(40.0)$ & $\begin{array}{l}2.667(1.039- \\
6.847)\end{array}$ & 0.0415 \\
\hline & No & $36(80.0)$ & $27(60.0)$ & & \\
\hline \multirow[t]{2}{*}{ ICU admission } & Yes & $23(51.1)$ & $28(62.2)$ & $\begin{array}{l}1.575(0.681- \\
3.648)\end{array}$ & 0.2886 \\
\hline & No & $22(48.9)$ & $17(37.8)$ & & \\
\hline \multirow[t]{2}{*}{ Thrombotic events } & Yes & $10(22.2)$ & $15(33.3)$ & $\begin{array}{l}1.750(0.686- \\
4.467)\end{array}$ & 0.2418 \\
\hline & No & $35(77.8)$ & $30(66.7)$ & & \\
\hline \multirow[t]{2}{*}{ Positive culture } & Yes & $3(10.0)$ & $4(9.5)$ & $\begin{array}{l}0.947(0.196- \\
4.582)\end{array}$ & 0.9464 \\
\hline & No & $27(90.0)$ & $38(90.5)$ & & \\
\hline $\begin{array}{l}\text { Days of mechanical } \\
\text { ventilation }\end{array}$ & $\begin{array}{l}\text { Median } \\
\text { (IQR) }\end{array}$ & $5(4-6)$ & $8(5-12)$ & n.a & 0.0213 \\
\hline $\begin{array}{l}\text { Days of non-invasive } \\
\text { ventilation }\end{array}$ & $\begin{array}{l}\text { Median } \\
\text { (IQR) }\end{array}$ & $3(2-4)$ & $3.5(1-6)$ & n.a & 0.7928 \\
\hline $\begin{array}{l}\text { Length of hospital stay, } \\
\text { days }\end{array}$ & $\begin{array}{l}\text { Median } \\
\text { (IQR) }\end{array}$ & $\begin{array}{l}13.5(9.8- \\
17)\end{array}$ & $\begin{array}{l}15(10- \\
19.5)\end{array}$ & n.a & 0.1304 \\
\hline
\end{tabular}

\section{Abbreviations}




\begin{tabular}{|c|c|}
\hline Abbreviation & Meaning \\
\hline COVID-19 & Coronavirus disease 2019 \\
\hline PCT & Procalcitonin \\
\hline OR & Odds Ratio \\
\hline $\mathrm{Cl}$ & Confidence Interval \\
\hline $\mathrm{p}$ & p-value \\
\hline WHO & World Health Organisation \\
\hline ICU & Intensive Care Unit \\
\hline CRP & C-Reactive Protein \\
\hline LDH & Lactate Dehydrogenase \\
\hline PCR & Polymerase Chain Reaction \\
\hline URT & Upper Respiratory Tract \\
\hline IM & Immunoassay \\
\hline MREC & Medical Research Ethics Committee \\
\hline ROC & Receiver Operative Characteristic \\
\hline IBM & International Business Machines Corporation \\
\hline SPSS & Statistical Package for the Social Sciences \\
\hline SD & Standard Deviation \\
\hline HIV & Human Immunodeficiency Virus \\
\hline AIDS & Acquired Immunodeficiency Syndrome \\
\hline NIV & Non-invasive Ventilation \\
\hline IQR & Interquartile Range \\
\hline CT scan & Computed Tomography \\
\hline AUC & Area Under Curve \\
\hline TP & True Positive \\
\hline FN & False Negative \\
\hline FP & False Positive \\
\hline TN & True Negative \\
\hline SPO2 & Oxygen saturation \\
\hline
\end{tabular}

Page 22/27 


\begin{tabular}{|ll|}
\hline GCS & Glasgow Coma Scale \\
\hline ANC & White Blood Cells \\
DM & Absolute Neutrophil Count \\
RR & Diabetes Mellitus \\
MV & Respiratory Rate \\
HPT & Mechanical Ventilation \\
\hline RA & Hypertension \\
\hline IL & Room Air \\
\hline IP-10 & Interleukin \\
\hline G-CSF & Granulocyte colony-stimulating factor \\
\hline TNF-alpha & Tumour Necrosis Factor alpha \\
\hline IFN-gamma & Interferon gamma \\
\hline
\end{tabular}

\section{Declarations}

\section{Ethics approval}

The study was approved by the Medical Research Ethics Committee (MREC), Ministry of Health, Malaysia, on April 6, 2021 (NMRR-21-458-59017) and (KKM/NIH/P21-564(4)). All methods were performed in accordance with the relevant guidelines and regulations. Consent to participate was waived as the study analyzed data retrospectively.

\section{Consent for publication}

The authors approved the final version of the manuscript and agreed to publication.

\section{Availability of data and materials}

The datasets generated and analyzed during the current study are available in the GitHub repository, https://github.com/william81/procalcitonin

\section{Competing interests}

The authors declared that no competing interest exists.

\section{Funding}


This study was funded by Siemens Healthcare Diagnostics for the article processing fee in an openaccess journal but the funder has no role in the design, conduct, data analysis, interpretation of findings, and manuscript preparation of the study.

\section{Authors' contributions}

C.W.T, D.K.Y.K, N.S.B.A.N, S.S, and K.C.L conceived and designed the study. C.W.T, D.K.Y.K, N.S.B.A.N and S.S collected the data. K.B.L and K.C.L carried out the analysis and interpretation of the results. S.K.A contributed to the funding acquisition. C.W.T, D.K.Y.K, K.B.L, N.S.B.A.N, S.S, K.C.L, S.K.A, S.N.A.B.S.B, and S.K.C contributed to writing, review, and/or revision of the manuscript.

\section{Acknowledgements}

The authors would like to thank the Director-General of Health for the approval to publish the research findings.

\section{References}

1. Xu, J. et al. Associations of procalcitonin, C-reaction protein and neutrophil-to-lymphocyte ratio with mortality in hospitalized COVID-19 patients in China. Scientific Reports10, 15058 (2020).

2. WHO Coronavirus (COVID-19) Dashboard. https://covid19.who.int/.

3. Ministry of Health Malaysia. (2020). Clinical Management of Confirmed COVID-19 Case in Adult. Retrieved from https://covid-19.moh.gov.my/garis-panduan/gp-umum-covid19.

4. Wu, C. et al. Risk Factors Associated With Acute Respiratory Distress Syndrome and Death in Patients With Coronavirus Disease 2019 Pneumonia in Wuhan, China. JAMA Internal Medicine180, 934-943 (2020).

5. Tahtasakal, C. A. et al. Could we predict the prognosis of the COVID-19 disease? Journal of Medical Virology $93,2420-2430$ (2021).

6. Allison, J., Hall, L., Maclntyre, I. \& Craig, R. K. The construction and partial characterization of plasmids containing complementary DNA sequences to human calcitonin precursor polyprotein. Biochem J199, 725-731 (1981).

7. Assicot, M. et al. High serum procalcitonin concentrations in patients with sepsis and infection. Lancet341, 515-518 (1993).

8. Rhodes, A. et al. Surviving Sepsis Campaign: International Guidelines for Management of Sepsis and Septic Shock: 2016. Intensive Care Medicine43, 304-377 (2017).

9. Cen, Y. et al. Risk factors for disease progression in patients with mild to moderate coronavirus disease 2019-a multi-centre observational study. Clin Microbiol Infect26, 1242-1247 (2020).

10. Li, J. et al. Meta-analysis investigating the relationship between clinical features, outcomes, and severity of severe acute respiratory syndrome coronavirus 2 (SARS-CoV-2) pneumonia. Am J Infect Contro/49, 82-89 (2021). 
11. Lippi, G. \& Plebani, M. Procalcitonin in patients with severe coronavirus disease 2019 (COVID-19): A meta-analysis. Clin Chim Acta505, 190-191 (2020).

12. Han, J. et al. Serum Procalcitonin Levels on Admission Predict Death in Severe and Critical COVID-19 Patients in Wuhan, China. Cardiovascular Innovation and Applications5, 37-44 (2020).

13. Guan, W.-J. et al. Clinical Characteristics of Coronavirus Disease 2019 in China. N Engl J Med382, 1708-1720 (2020).

14. Slaats, J., ten Oever, J., van de Veerdonk, F. L. \& Netea, M. G. IL-1ß/IL-6/CRP and IL-18/ferritin: Distinct Inflammatory Programs in Infections. PLOS Pathogens12, e1005973 (2016).

15. Guo, J. et al. Cytokine Signature Associated With Disease Severity in COVID-19. Frontiers in Immunology12, 3276 (2021).

16. Manufacturer Insert Procalcitonin (PCT) on Atellica IM 11202687_EN Rev.03, 2019-07.

17. R Core Team. A language and environment for statistical computing. R Foundation for Statistical Computing, Vienna, Austria. (2021).

18. Ho, D., Imai, K., King, G. \& Stuart, E. A. Matchlt: Nonparametric Preprocessing for Parametric Causal Inference. J. Stat. Soft.42, 1-28 (2011).

19. Thiele, C. \& Hirschfeld, G. cutpointr: Improved Estimation and Validation of Optimal Cutpoints in R. J. Stat. Soft.98, 1-27 (2021).

20. Vanhomwegen, C. et al. Procalcitonin accurately predicts mortality but not bacterial infection in COVID-19 patients admitted to intensive care unit. Ir J Med Sci 1-4 (2021) doi:10.1007/s11845-02002485-z.

21. Tang, J. et al. Serum IL-6 and procalcitonin are two promising novel biomarkers for evaluating the severity of COVID-19 patients. Medicine100, (2021).

22. Hu, R., Han, C., Pei, S., Yin, M. \& Chen, X. Procalcitonin levels in COVID-19 patients. Int J Antimicrob Agents56, 106051-106051 (2020).

23. An, P.-J., Zhu, Y. Z. \& Yang, L.-P. Biochemical indicators of coronavirus disease 2019 exacerbation and the clinical implications. Pharmacol Res159, 104946-104946 (2020).

24. Kamat, I. S., Ramachandran, V., Eswaran, H., Guffey, D. \& Musher, D. M. Procalcitonin to Distinguish Viral From Bacterial Pneumonia: A Systematic Review and Meta-analysis. Clinical Infectious Diseases70, 538-542 (2020).

25. Moore John B. \& June Carl H. Cytokine release syndrome in severe COVID-19. Science368, 473-474 (2020).

26. Que, Y. et al. Cytokine release syndrome in COVID-19: a major mechanism of morbidity and mortality. Int Rev Immunol 1-14 (2021) doi:10.1080/08830185.2021.1884248.

27. Ji, P. et al. Association of elevated inflammatory markers and severe COVID-19: A meta-analysis. Medicine (Baltimore)99, e23315-e23315 (2020).

28. Liu, F. et al. Prognostic value of interleukin-6, C-reactive protein, and procalcitonin in patients with COVID-19. J Clin Viro/127, 104370-104370 (2020). 
29. Huang, I., Pranata, R., Lim, M. A., Oehadian, A. \& Alisjahbana, B. C-reactive protein, procalcitonin, Ddimer, and ferritin in severe coronavirus disease-2019: a meta-analysis. Ther Adv Respir Dis14, 1753466620937175-1753466620937175 (2020).

30. Agarwal, A. et al. High-flow nasal cannula for acute hypoxemic respiratory failure in patients with COVID-19: systematic reviews of effectiveness and its risks of aerosolization, dispersion, and infection transmission. Can J Anaesth67, 1217-1248 (2020).

31. Demoule, A. et al. High-Flow Nasal Cannula in Critically III Patients with Severe COVID-19. Am J Respir Crit Care Med202, 1039-1042 (2020).

32. Attaway, A. H., Scheraga, R. G., Bhimraj, A., Biehl, M. \& Hatipoğlu, U. Severe covid-19 pneumonia: pathogenesis and clinical management. BMJ372, n436 (2021).

33. Ji, Y., Ma, Z., Peppelenbosch, M. P. \& Pan, Q. Potential association between COVID-19 mortality and health-care resource availability. The Lancet Global Health8, e480 (2020).

34. Emanuel, E. J. et al. Fair Allocation of Scarce Medical Resources in the Time of Covid-19. N Engl J Med382, 2049-2055 (2020).

35. Pham, T., Brochard, L. J. \& Slutsky, A. S. Mechanical Ventilation: State of the Art. Mayo Clinic Proceedings92, 1382-1400 (2017).

\section{Figures}


Patients with PCR confirmed COVID-19 admitted to Sungai Buloh Hospital in December 2020 and had their PCT measured $(n=482)$

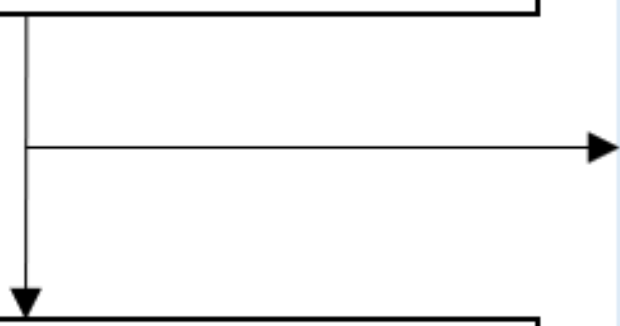

Patients who fulfilled inclusion criteria and were included in this study $(\mathrm{n}=319)$

\section{Inclusion criteria}

- Age above 18 years old

- Patients' diagnosis of COVID-19 via PCR test

- Have PCT level measured during hospitalization

- Category 4 and 5

\section{Exclusion from analysis}

- Patients with PCT level $>100$ $\mathrm{ng} / \mathrm{mL}$ as outlier $(\mathrm{n}=3)$

- Patients who had their PCT level measured after 5 days of admission $(n=40)$

\section{Level 1 optimal cut-point analysis}

Patients with PCT levels measured within 5 days following admission $(n=276)$

Patients who were included in the analysis of optimal cut-point for PCT $(\mathrm{n}=276)$

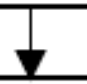

\section{Level 2 optimal cut-point analysis}

Patients with PCT levels measured within 3 days following admission $(n=237)$

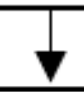

\section{Propensity score matching}

Patients who were matched for covariate differences $(n=90)$

\section{Figure 1}

Flowchart of patient recruitment 